\section{Podoconiosis, a neglected lymphatic tropical disease}

\section{Paolo Zamboni, Mirko Tessari \\ HUB Center for Venous and Lymphatic Diseases, AOU of Ferrara, Italy}

\begin{abstract}
Podoconiosis causes a painful massive swelling of the lower limbs, bilaterally and asymmetrically. It is caused by exposure to particles common in soils of volcanic origin and is second only to lymphatic filariasis as the leading cause of tropical lymphoedema. An estimated 4 million people live with podoconiosis globally in 32 potentially endemic countries. Podoconiosis is associated with positive family history of podoconiosis, bare foot, gender, poor housing condition, foot hygiene, income and educational status of the affected patients. There are also cultural barriers involved in maintaining a high epidemiology of the disease. Podoconiosis was never been prioritized either in intervention or research programmes. This may be due to the lack of resources for new health initiatives, which is a common problem in the low-income tropical countries in which this disease is present. Only Ethiopia, Cameroon, and Rwanda report podoconiosis within their routine health management information systems.We believe that comprehensive podoconiosis control strategies such as promotion of footwear and personal hygiene are urgently needed in endemic countries in the African Region. Mapping, active surveillance and a systematic approach to the monitoring of disease burden must accompany the implementation of podoconiosis control activities.
\end{abstract}

\section{Definition and epidemiology}

Until 20 years ago, podoconiosis was practically not described in medical literature, and the sufferers was a private matter that was not dealt with by the formal heath sector. $^{1}$

Podoconiosis causes a painful massive swelling of the lower limbs, bilaterally and asymmetrically. It is caused by exposure to particles common in soils of volcanic origin and is second only to lymphatic filariasis as the leading cause of tropical lymphoedema. An estimated 4 million people live with podoconiosis globally in 32 potentially endemic countries. ${ }^{2}$

Podoconiosis is found in highland areas of tropical Africa, Central America and north-west India. In Figure 1 it well apparent the global distribution of podoconiosis. ${ }^{3}$

Despite the significance of the disease, its global distribution and epidemiology are poorly understood, according to WHO document where is reported that in many endemic countries prevalence and incidence were never investigated. ${ }^{2}$ A systematic review assessed the epidemiology of podoconiosis in 18 endemic coutries from Africa, 3 from Asia and 11 from Latin America.

None of the Latin American countries reported prevalence data, although some countries are suspected to be endemic. ${ }^{3}$

In 2011 the World Health Organization (WHO) recognized podoconiosis as one of the neglected tropical diseases (NTDs) under the category of 'other tropical conditions'. ${ }^{2}$ Subsequently Ethiopia, Rwanda and Cameroon recognized podoconiosis as a priority NTD and included it in their longterm health plans, with scarce results, yet.

\section{The peculiarity of podoconiosis}

Podoconiosis is really a different disease respect to lymphedema in the Western Countries or to lymphatic filariasis in the tropical regions. Podoconiosis is caused by exposure to red clay soil, and results from a classic interaction between genes and environmental factors. Particularly, mineral particles from the soil penetrate the skin and are taken up by macrophages in the lymphatic system which causes inflammation and fibrosis of the vessel lumen leading to blockage of the lymphatic drainage. This results in oedematous feet and legs and subsequently progresses to elephantiasis and fibrous-edema. These changes are themselves disabling, and painful intermittent acute lymphangitis episodes cause further aggravation (Figure 2). ${ }^{4}$

There is lack of knowledge regarding the pathogenesis of the disease because it is still unclear why podoconiosis strikes not all the people who does not foot wear in the endemic areas. Podoconiosis was associated with positive family history of podoconiosis, bare foot, gender, poor housing condition, foot hygiene, income and educational status of the affected patients. ${ }^{5}$

Individuals with family history of podoconiosis were 2.81 times more likely to develop podoconiosis than individuals with no family history of podoconiosis [AOR, 2.81 (95\% CI: 1.7-4.64)]. This finding suggests the presence of unknown susceptibility genes.

The probability of podoconiosis in barefooted individuals was 3.26 times higher
Correspondence: Paolo Zamboni, Chair HUB Center for Venous and Lymphatic Diseases, Regione Emilia Romagna, Azienda Ospedaliera Universitaria di Ferrara, Ferrara, Italy.

Tel.: +39.0532237694

E-mail: paolozamboni@icloud.com

Key words: Podoconiosis; leg swelling; edema; lymphedema; neglected tropical disease.

Acknowledgments: The authors thank Mr. Filippo Guidi BSc, Director of StreetSpirit NGO operating in Ethiopia and Uganda, for the precious enviromental and cultural information.

Received for publication: 30 January 2020. Revision received: 14 February 2020.

Accepted for publication: 14 February 2020

This work is licensed under a Creative Commons Attribution 4.0 License (by-nc 4.0)

(C) Copyright: the Author(s), 2020

Licensee PAGEPress, Italy

Veins and Lymphatics 2020; 9:8859

doi:10.4081/vl.2020.8859

than in individuals who wear shoe in their daily activities [AOR, 3.26 (95\% CI: 2.035.25)]. This finding supports that barefooted patients individuals may allow soil substances to enter into their body, initiating the pathophysiology of the disease.

The likelihood of the disease was found 3.85 times higher in the male gender, maybe due to different exposure distribution (working activities?).

The risk of acquiring podoconiosis in people living in poor housing conditions was $83 \%$ higher [AOR, 0.17 (95\% CI: 0.1$0.3)$ ], supporting the role of personal hygiene. In fact the probability of the diseases in an individual who did not frequently keep his/her foot hygiene was 2.68 times higher than with an individual that keep his/her foot hygiene in their daily life [AOR, 2.68 (95 CI: 1.72 - 4.19)]. A further confirm is the $88 \%$ increased risk of podoconiosis in patients with low income [AOR, 0.12 (95\% CI: 0.07-0.22)].

Finally, as compared to the illiterate, the risk of podoconiosis was $98 \%$ lower with primary education [AOR, 0.02 (95\% CI: $0.01-0.04)], 99 \%$ for secondary education [AOR, 0.01 (95\% CI: 0.005-0.02)] while tertiary education decreased the risk up to 99.97\% [AOR, 0.003 (95\% CI: 0.0010.007)]. This finding supports that educational status increases awareness on foot hygiene.

One more peculiarity of podoconiosis in the endemic countries is linked to significant 
stigma, discrimination and comorbid mental health problems, including a significant number of people with depression. ${ }^{6,7}$ The disease has a significant impact in reducing the economic productivity of affected individuals and their families. ${ }^{8}$ All these justify the need for recognition of podoconiosis as a public health challenge and the development of a comprehensive and adequately resourced global health strategy towards elimination of podoconiosis.

\section{The cultural barriers and their consequences}

In southern Ethiopia, shoes are being distributed through a local non-governmental organization to children with the intention of preventing podoconiosis. It seems obvious that wearing shoes may help in preventing onset or progression of podoconiosis, but also of a wide range of NTDs. A study assessed the factors that influenced shoe wearing behaviors among people living in a rural community in highland Ethiopia. ${ }^{9}$

The authors conducted structured individual interviews, focus group discussions and extended case studies among 242 adults and systematically analyzed the information. They found that shoe wearing is intermittent, and that different factors such as cost and ability to use the shoes for certain activities (such as farming) influenced consistent shoe wearing for most people. Some factors (such as shoe size, fear of stigma) were more relevant for podoconiosis patients. Interestingly, children exhibited greater desire to wear shoes than adults.

A community leader of 50 y.o. stated that people cannot wear shoes everywhere due to lack of alternative pairs of shoes. They wear it economically only when they go to places like funerals, weddings, churches, market and other faraway places. They fear that the shoe might get old if they wear it in the farm, in the rain or performing other household activities. Some people even take off shoes while it is raining for fear it may destroy their shoes. When going to town, some people take off their shoes if the road is muddy and put it on when they reach the dry roads. ${ }^{9}$

Such cultural barriers have of course relevance for preventing and treat podoconiosis in the endemic countries.

As the disease progresses, podoconiosis patients became bedridden, isolated from the community activities. ${ }^{10}$ All podoconiosis patients did not perform their daily work properly; as a result of this, they had low income. Annually, Ethiopia losses more than 200 million USA dollars due to podoconiosis. ${ }^{11-13}$

\section{Can we defeat podoconiosis?}

The above reported lack of epidemiologic data in several countries, is mainly due to the absence of accurate and easy-touse diagnostic tools such as a point-of-care diagnostic test. Currently, diagnosis is clinical and based on exclusion of other potential causes of lymphedema in the tropics, mostly lymphatic filariasis but also certain forms of leprosy, Milroy syndrome, heart or liver disfunction, glomerulonephritis, and intestinal malabsorption. ${ }^{6}$

Nonetheless, in endemic areas trained health workers can easily identify the disease, and educational activity needs to be associated in order to eliminate the cultural barriers, and to increase the awareness of foot hygiene and shoes wearing.

Podoconiosis was never been prioritized either in intervention or research programmes. This may be due to the lack of resources for new health initiatives, which is a common problem in the low-income tropical countries in which this disease is present. Only Ethiopia, Cameroon, and Rwanda report podoconiosis within their routine health management information systems. ${ }^{13}$

Since water is a scarce resource in the endemic area, a randomized control trial was conducted over 3 months on 193 patients. The intervention arm comprised $2 \%$ glycerine $(\mathrm{v} / \mathrm{v})$ added to a reduced amount of soaking water. The control group received the current skin care regimen. Primary outcome measures were trans-epidermal water loss (TEWL) and stratum corneum hydration ( $\mathrm{SCH})$. Improvement in SBF was observed in both groups across all measurement sites and time points, although this was significantly greater in the experimental group. TEWL reduced in both groups at all sites but more significantly in the intervention arm. Finally, there were also significantly greater reductions in odor, number of wounds and largest foot circumference in the experimental compared to the control group. ${ }^{14}$

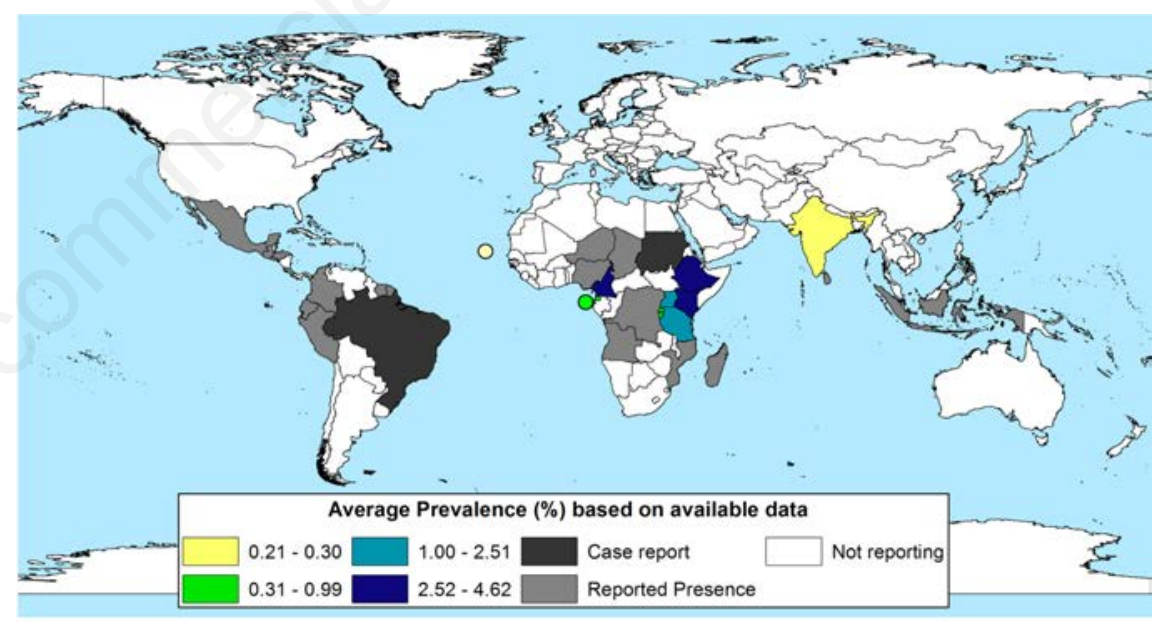

PLoS Negl Trop Dis, 2018, 12(3): e0006324.

Figure 1. Global epidemiology of podoconiosis. Modified from Deribe et al., 2018. ${ }^{3}$

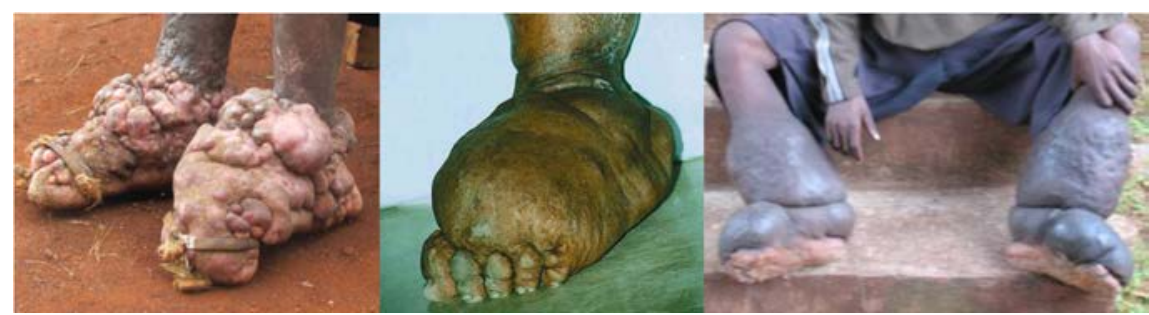

Figure 2. Clinical presentation of advanced cases of podoconiosis. 
We believe that comprehensive podoconiosis control strategies such as promotion of footwear and personal hygiene are urgently needed in endemic countries in the African Region. Mapping, active surveillance and a systematic approach to the monitoring of disease burden must accompany the implementation of podoconiosis control activities.

Advocacy is critical to improve the awareness of the disease by decision makers. Identifying public figures as podoconiosis testimonials is mandatory to achieve this goal. The use of social media and foundations to increase public awareness is important. The first international conference on podoconiosis was held in 2018 in Addis Ababa, Ethiopia, claiming a call for global action. The awareness of the disease should reach out to donors, policymakers and program planners.

A good proposal could be to promote a pilot study in a little area, experimenting a strategic program. If the latter will demonstrate to be efficient, it should be used as a global model of intervention.

\section{Treatment modalities}

Apart the etiology and the other peculiarities around and behind podoconiosis, the disease remains basically a lower limb lymphedema. For such a reason, out of prevention program, the patients who are affected by podoconiosis need of the standard treatment for lymphedema. Obviously, compression, with bandages, is the cornerstone of the treatment. However, in the affected areas there is a lack of specialized out patients clinics to have a regular bandaging treatment. Moreover, for the irregular morphology of the lower extremities, as well as for the different disease stage in the affected patients (see a gallery of images at https://www.bing.com/images/search?q=po doconiosis\&FORM=HDRSC2), it might be proposed to use adjustable compression wrap devices (ACWD). The latter show obvious advantages if an education program should be available on site. ACWD advantages include self-management, self-application, re-adjustability and standardization of the compression therapy.

Basically, all systems are one or more low stretch bandages that encircle the lower leg and adhere to itself with velcro. Some allow for a selective adjustment during the course of the application because in these systems the individual bands mutually intermesh. In others, the bandages must always be opened from proximal to distal in order to retighten individual segments.

Furthermore, it seems to improve the compliance of the patients to compression treatment. ${ }^{15-18}$

Finally, skin and wound care for lesions and/or hyperkeratosis, need to be performed on outpatient basis according to standard therapeutic principles, when appropriate.

\section{References}

1. Deribe K, Tomczyk S, Tekola-Ayele F. Ten years of podoconiosis research in Ethiopia. PLoS Negl Trop Dis 2013;7:e2301.

2. World Health Organization. Podoconiosis: endemic non-filarial elephantiasis. Available from: http://www. who.int/lymphatic filariasis/epidemiology/podoconiosis/en/ Accessed :17 January 2018.

3. Deribe K, Cano J, Trueba ML, et al. Global epidemiology of podoconiosis: A systematic review. PLoS Negl Trop Dis 2018;12:e0006324.

4. Davey G, Tekola F, Newport MJ. Podoconiosis: non-infectious geochemical elephantiasis. Trans R Soc Trop Med Hyg 2007;101:1175-80.

5. Berhanu E. Determinants of Podoconiosis: A Case Control Study. J Health Sci 2017;27:501.

6. Sime H, Deribe K, Assefa A, et al. Integrated mapping of lymphatic filariasis and podoconiosis: lessons learnt from Ethiopia. Parasit Vectors 2014;7: 397.

7. Bartlett J, Deribe K, Tamiru A, et al. Depression and disability in people with podoconiosis: a comparative cross-sectional study in rural northern Ethiopia. Int Health 2015;8:124-31.

8. Tekola F, Haile MD, Davey G.
Economic costs of endemic nonfilarial elephantiasis in Wolaita Zone, Ethiopia. Trop Med Int Health 2006;11:1136-44.

9. Ayode D, McBride CM, de Heer HD, et al. A qualitative study exploring barriers related to use of footwear in rural highland Ethiopia: implications for neglected tropical disease control. PLoS Negl Trop Dis 2013;7:e2199.

10. Tekola F, Mariam D, Davey G. Economic costs of endemic non-filarial elephantiasis in Wolaita Zone, Ethiopia. Trop Med Int Health 2006;11:1136-44.

11. Destas K, Ashine M, Davey G. Prevalence of podoconiosis (endemic nonfilarial elephantiasis) in Wolaitta, South Ethiopia Trop Doct 2003;33:21720.

12. Yordanos B, Sara T, Tsige A, et al. Patients' perceptions of podoconiosis causes, prevention and consequences in East and West Gojam, Northern Ethiopia. BMC Public Health 2011;12: 828.

13. Deribe K, Wanji S, Shafi O, et al. The feasibility of eliminating podoconiosis. Bull World Health Org 2015;93:712-8.

14. Brooks J, Ersser SJ, Cowdell F, et al. A randomized controlled trial to evaluate the effect of a new skincare regimen on skin barrier function in those with podoconiosis in Ethiopia. Br J Dermatol 2017;177:1422-31.

15. Kroeger K, Dissemond J. Chronic venous insufficiency and interest of adjustable compression wrap devices. Veins and Lymphatics 2019;8:8054.

16. Weller CD, Buchbinder R, Johnston RV. Interventions for helping people adhere to compression treatments for venous leg ulceration. Cochrane Database Syst Rev 2016;3:CD008378.

17. Stansal A, Tella E, Yannoutsos A, et al. Supervised short-stretch compression therapy in mixed leg ulcers. J Med Vasc 2018;43:225-30.

18. Bender D, Kuhn PJ, Olson DJ, Sullivan JP. Adjustable topical compression foot wrap, is more effective than a dopamine agonist, ropinirole, in reducing the symptoms of moderate to severe restless leg syndrome. Veins and Lymphatics 2016;5:5994. 